\title{
The impact of integrating medical assistants and community health workers on diabetes care management in community health centers
}

Hector P. Rodriguez ${ }^{1 *}$ (D, Mark W. Friedberg ${ }^{2}$, Arturo Vargas-Bustamante ${ }^{3}$, Xiao Chen ${ }^{4}$, Ana E. Martinez ${ }^{4}$ and Dylan H. Roby ${ }^{5}$

\begin{abstract}
Objective: To compare the impact of implementing team-based diabetes care management involving community health workers (CHWs) vs. medical assistants (MA) in community health centers (CHCs) on diabetes care processes, intermediate outcomes, and patients' experiences of chronic care.

Data sources: Clinical and administrative data $(n=6111)$ and patient surveys $(n=698)$ pre-intervention and postintervention. Surveys $(n=285)$ and key informant interviews $(n=48)$ of CHC staff assessed barriers and facilitators of implementation.

Study design: A three-arm cluster-randomized trial of $\mathrm{CHC}$ sites integrating MAs $(n=3)$ or CHWs $(n=3)$ for diabetes care management compared control CHC sites $(n=10)$. Difference-in-difference multivariate regression with exact matching of patients estimated intervention effects.

Principal findings: Patients in the CHW intervention arm had improved annual glycated hemoglobin testing $(18.5 \%, p<0.001)$, while patients in the MA intervention arm had improved low-density lipoprotein cholesterol control $(8.4 \%, p<0.05)$ and reported better chronic care experiences over time $(\beta=7.5, p<0.001)$. Except for chronic care experiences $(p<0.05)$ for patients in the MA intervention group, difference-in-difference estimates were not statistically significant because control group patients also improved over time. Some diabetes care processes improved significantly more for control group patients than intervention group patients. Key informant interviews revealed that immediate patient care issues sometimes crowded out diabetes care management activities, especially for MAs.
\end{abstract}

Conclusions: Diabetes care improved in CHCs integrating CHWs and MAs onto primary care teams, but the improvements were no different than improvements observed among matched control group patients. Greater improvement using CHW and MA team-based approaches may be possible if practice leaders minimize use of these personnel to cover shortages that often arise in busy primary care practices.

Keywords: Community health centers, Medical assistants, Community health workers, Diabetes care management, Patients' experiences, Implementation research

\footnotetext{
* Correspondence: hrod@berkeley.edu

${ }^{1}$ Division of Health Policy and Management, School of Public Health,

University of California, Berkeley, 50 University Hall, Room 245, Berkeley, CA

94720, USA

Full list of author information is available at the end of the article
}

(c) The Author(s). 2018 Open Access This article is distributed under the terms of the Creative Commons Attribution 4.0 International License (http://creativecommons.org/licenses/by/4.0/), which permits unrestricted use, distribution, and reproduction in any medium, provided you give appropriate credit to the original author(s) and the source, provide a link to the Creative Commons license, and indicate if changes were made. The Creative Commons Public Domain Dedication waiver (http://creativecommons.org/publicdomain/zero/1.0/) applies to the data made available in this article, unless otherwise stated. 


\section{Background}

The effectiveness of team-based models of primary care that include registered nurse care management [1] and pharmacist-led medication management [2] in improving diabetes care quality and patient self-management is well established. Despite evidence of effectiveness, these models for managing diabetes care are not financially feasible for many community health centers (CHCs) that serve low-income patients [3]. Team-based models that involve patient panel management by medical assistants (MAs) and/or community-based care management by community health outreach workers (CHWs) are relatively more financially feasible for $\mathrm{CHCs}$ to adopt and implement. Despite the large disease burden of diabetes among vulnerable populations [4,5], the effect of these lower cost and scalable team-based approaches on improving the diabetes care management remains unclear.

Previous research has demonstrated the patient health benefits of diabetes education, especially programs and interventions that emphasize patient self-management for vulnerable patients [6, 7], and MAs and CHWs can provide self-management support to diabetic patients and change patient knowledge and behavior and, in some instances, health outcomes [8-10].

MAs are projected to be among the fastest growing occupational groups between 2016 and 2026 [11] and are the fastest growing group on adult primary care teams [12]'. Demand for MA support and services in health coaching and panel management roles is likely to increase as a result of changing external incentives toward value-based payment [13]. The potential positive impact of MA health coaching and panel management interventions on patient outcomes and experiences of care in underserved settings highlights the promise for expanding MA responsibilities to support diabetes care management [14-17].

CHWs also have competencies that enable them to support patients with diabetes. They are peer educators whose goal is to promote health in their community through information distribution, assistance, social support, and organizing community networks [18, 19]. CHW roles have included: member of care delivery teams, navigator, screening and health education provider, outreach and enrolling agent, and organizer [9]. CHWs generally work with patients in community settings and some conduct home visits or support groups. CHWs can improve diabetes self-management skills among patients [20]. However, few studies have assessed the effectiveness of CHWs in improving outcomes of care in routine settings [21, 22], and while several patient-level randomized controlled trials have been conducted [23], we know of no studies that have examined the effectiveness of routine implementation of CHWs in $\mathrm{CHCs}$ using a cluster randomized research design.
We compared the impact of implementing CHWs and MA with expanded roles and responsibilities for managing diabetes care in $\mathrm{CHCs}$ that care for predominantly low-income Latino Spanish-speaking or Chinese patients. To our knowledge, no research has compared the impact of integrating MAs vs. CHWs as members of $\mathrm{CHC}$ primary care teams compared to a control group. We hypothesized that MA panel management and $\mathrm{CHW}$ integration would have different impacts on diabetes care management. Because MAs are office-based and focus on ensuring that patients receive appropriate treatments and tests [13], we hypothesized that team-based models of diabetes care management involving MAs would improve process of care measures to a greater degree than teams with CHWs. In contrast, CHWs' roles focus on patient activation and self-management in community settings [9], where individuals make important diet and lifestyle choices. As a result, their contributions to diabetes management may be more likely to result in patient engagement and self-management compared to MAs. Given the challenge of measurably improving patients' experiences [24], we anticipated that changes in patients' experiences of care resulting from implementing the team-based models would be modest and similar for sites integrating $\mathrm{CHW}$ and MAs.

\section{Methods}

Study design

A cluster-randomized trial using effectiveness-implementation Type II study design [25] was conducted with the simultaneous goals of examining the impact of integrating MAs or CHWs into care teams on patient outcomes and assessing barriers and facilitators of routine integration of new care team members, roles, and responsibilities. We recruited $16 \mathrm{CHC}$ sites located in three counties in Northern California that were all affiliated with the same regional community clinic association. The participating $\mathrm{CHCs}$ served primarily low-income, Latino and Chinese patients and patients. All CHC sites studied have bilingual and bicultural staff that can provide care in patients' language preference.

Prior to site randomization from June-August 2011, we conducted practice surveys of each site director $(n=15$, response rate: 94\%) and 281 adult primary care clinicians and staff members from each participating $\mathrm{CHC}$ (response rate: $81 \%$ ) to assess baseline practice climate and organizational factors that would impact the effectiveness of clinic efforts to implement team-based models of care. The site director survey assessed the diabetes care management capabilities of $\mathrm{CHC}$ sites, including use of registries, self-management education, use of data for improvement purposes, and connection to community resources [26]. A 
validated survey of $\mathrm{CHC}$ adult primary care clinicians and staff was used to assess teamwork and practice climate [27].

Using the response of the site director and mean responses to the clinician and staff surveys, we dichotomized practices into "high" (top eight) vs. "low" (bottom eight) on each of the following composite measures: 1) diabetes structural capabilities, 2) primary care team functioning, 3) practice size (mean number of clinicians/staff: 18). Using cluster analyses, we grouped practices into three sampling strata based on the most common combinations of these three composite measures and randomly assigned practices within each strata to the MA intervention $(n=3)$, $\mathrm{CHW}(n=3)$, and control $(n=10)$ group arms of the cluster-randomized trial. Stipends equivalent to the costs of personnel and benefits of new MA and CHW team members were provided to each $\mathrm{CHC}$ intervention site for one year (2012). Each participating site received a stipend each year (2011-2013) to defray the costs of clinic and administrative data collection and reporting required of research study participation.

\section{Interventions}

In most cases (5 of 6), existing MAs were promoted to take on the new responsibilities for diabetes care management. All CHCs hired replacements for the MAs taking on the project roles. MA and CHW personnel from intervention $\mathrm{CHC}$ sites were trained on health coaching and panel management for diabetes care by expert trainers prior to the intervention, between October and December 2011, over three in-person six-hour sessions. Table 1 summarizes the roles and responsibilities for managing diabetes care emphasized in MA vs. CHW training and implementation. All intervention personnel received training in motivational interviewing. The MA training emphasized office-based panel management activities for diabetic patients, including maintaining the diabetes registry, reviewing the panel at regular intervals, following up on primary care clinician instructions, assisting with medication reconciliation, and targeting patients for appropriate interventions and referral to community resources. The MAs also received a brief module on health coaching strategies. In contrast, the

Table 1 A comparison of medical assistant and community health worker roles in managing diabetes care for training and implementation

\begin{tabular}{|c|c|c|c|}
\hline & $\begin{array}{l}\text { Medical assistant } \\
\text { intervention arm }\end{array}$ & $\begin{array}{l}\text { Community health worker } \\
\text { intervention arm }\end{array}$ & $\begin{array}{l}\text { Control group } \\
\text { arm }^{\mathrm{a}}\end{array}$ \\
\hline \multicolumn{4}{|l|}{ Pre-visit } \\
\hline Discuss the patient case with the physician & $x$ & & \\
\hline Agenda setting with the patient & $x$ & $x$ & \\
\hline Ordering routine services & $x$ & $x$ & $x$ \\
\hline History tracking & $x$ & & \\
\hline \multicolumn{4}{|l|}{ During the Visit } \\
\hline Document physician findings & $x$ & & \\
\hline Send electronic prescriptions to pharmacy & $x$ & & \\
\hline Write prescriptions for the physician to sign & $x$ & & \\
\hline \multicolumn{4}{|l|}{ Post-visit } \\
\hline Discuss patients' concerns & $x$ & $x$ & \\
\hline Recapitulate the advice given by the physician & $x$ & $x$ & \\
\hline Set goals with the patient & $x$ & $x$ & \\
\hline Make sure that patients can navigate the system & & $x$ & \\
\hline \multicolumn{4}{|l|}{ Between Visits } \\
\hline Provide culturally appropriate and accessible health education and information & $x$ & $x$ & \\
\hline Assure that people with diabetes receive the services they need & $x$ & $x$ & $x$ \\
\hline Follow up over the phone & $x$ & $x$ & \\
\hline Offer informal counseling and social support & & $x$ & \\
\hline $\begin{array}{l}\text { Provide information to families to support the lifestyle changes of patients with } \\
\text { diabetes }\end{array}$ & & $x$ & \\
\hline Build individual and community capacity & & $x$ & \\
\hline
\end{tabular}

${ }^{a}$ Community health center sites in the control group did not implement major changes to diabetes care processes during the study period, as verified by key informant interviews during the study period. Most control CHC sites, however, had some support for pre-visit ordering of routine diabetes services and follow up to assure services were received for patients in general (not specific to patients with diabetes) 
CHW training emphasized a broader range of patient health coaching skills, including helping patients set their agendas for clinicians visits, making sure patients understand what their clinicians want them to do, determine whether patients agreed with their care plans, provide support to patients' efforts in adopting healthy behaviors, assist patients to improve medication understanding and adherence [28]. The CHWs also received a brief, less extensive module on panel management activities. Once trained, the personnel followed role-specific diabetes care management protocols developed by the participating clinics' quality improvement teams in collaboration with the regional community clinic association.

Intervention $\mathrm{CHC}$ site administrators and frontline personnel received ongoing technical assistance from a regional community clinic association, including managing and reporting clinical and administrative data central to assessing diabetes care processes and intermediate outcomes, reinforcing motivational interviewing skills among MA and CHW staff, and didactic refresher trainings on evidence-based diabetes care management. The intervention period was from January-December 2012, but all practices continued to use the team-based models through 2013.

\section{Clinical and administrative data}

The patient analytic sample was defined as diabetic using ICD-9 codes 250, 357.2, 362.0, 366.41, and 648.0. To supplement missing information in encounter data, we used additional methods to identify patients with diabetes, including pharmacy, billing/claims, and other encounter record data. We analyzed patient-level information on demographic information, $\mathrm{CHC}$ visits, and clinical measures of blood pressure, low-density lipoprotein cholesterol (LDL-C), and glycated hemoglobin (HbA1c). Healthcare Effectiveness Data Information Set (HEDIS) comprehensive diabetes care measure definitions were used to construct dichotomous outcome measures of control: HbA1c $<8.0 \%$, blood pressure $<140 / 90 \mathrm{mmHg}$, and LDL-C $<100$ were considered "controlled".

\section{Patient experience surveys}

The early intervention patient experience survey of adult diabetics was fielded from July-August 2012 and the post-intervention survey of baseline respondents was fielded from July-August 2013. Surveys asked about care experiences during the prior six months. A total of 167 randomly sampled patients per control practice and up to 400 patients at each of the intervention practices were sampled for the survey. Sampled patients had at least 2 visits with a diabetes diagnosis during the pre-intervention year (2011). The survey was administered in English,
Spanish or Chinese and included a $\$ 10$ gift card. Patients were sent a survey in the mail with the gift card, and non-respondents received a second survey and then were called by phone with up to 8 attempts for each patient to complete the survey with an interviewer. Patients contacted by phone were consented by phone, while patients returning the survey consented by returning the survey. The pre-intervention $(n=1298$; RR $=47 \%)$ and post-intervention $(n=698 ; \mathrm{RR}=65 \%)$ survey responses were merged to generate the cohort for the survey analyses.

Survey questions included the Clinician \& Group Consumer Assessment of Healthcare Providers and System (CG-CAHPS) survey [29], Patient Assessment of Chronic Illness Care (PACIC-11) measure [30], the Problem Areas in Diabetes (PAID-5) scale [31], and a count of self-reported hypoglycemic events over the past month. [32] Like many CHCs nationally, the sampled $\mathrm{CHCs}$ did not empanel patients to specific primary care clinicians (PCCs) due to high PCC turnover [33] and instead assigned patients to teams or to the entire practice. Accordingly, we adapted the standard CG-CAHPS questions to reference "doctors and nurses at this clinic" rather than "your personal doctor". PACIC-11 is widely used to evaluate the delivery of chronic care management activities for a variety of conditions, including diabetes [30, 34]. PAID-5 is a psychometrically robust short-form measure of diabetes-related emotional distress [31]. Finally, a hypoglycemia measure was included because it was an important adverse event and contributor to poorer diabetes outcomes [32]. We used the half-scale rule [35] to calculate composites scores, whereby respondents had to complete at least half of the items comprising the composite measure for a score to be calculated. For ease of interpretation, we transformed the unweighted average of the survey composite questions to a 0 to 100 scale when calculating composite scores.

\section{Key informant interviews}

Mid-intervention semi-structured interviews of $\mathrm{CHC}$ clinician and staff key informants were conducted from May-August 2012 and post-intervention interviews of the same key informants were conducted between March-June 2013. A total of 48 interviews were conducted with 28 individual clinicians and staff who provided consent to participate. This approach allowed for in-depth data collection to identify changes in diabetes care processes, and assess facilitators and barriers of implementation. Although the control sites were not encouraged to implement any changes, we conducted interviews to understand any changes to diabetes care processes and capabilities to shed light on any over-time changes in diabetes care processes among 
intervention clinics. All interviews were conducted by telephone or in-person using an interview guide and were audio recorded and transcribed. The aim of the interviews was to learn about changes in diabetes care at the $\mathrm{CHC}$ site, and perceived barriers and facilitators of implementing the team-based approach involving MAs and/or CHWs [36]. Informed consent for recording and transcribing the interviews was obtained for all participants at the beginning of each interview. Interview participants received a $\$ 25 \mathrm{gift}$ card for participating in each interview. The human research ethics committee at University of California Los Angeles approved the study protocol (IRB\#10000596).

\section{Analytic samples}

Data collection and reporting was complicated by the diverse clinical information and administrative systems being queried at each of the sites to supply the patient-level data. $\mathrm{CHCs}$ have high proportions of patients with unstable home and work environments that impact their ability to follow-up and manage their diabetes. In our $\mathrm{CHC}$ patient population, a total of 11,249 unique adult diabetic patients had at least one visit with a diabetes diagnosis at one of the $14 \mathrm{CHC}$ sites in 2011. Since we used the SUPREME-DM definition [37] to define our analytic sample, we included patients with 2 or more visits, excluding 4163 (37.0\%) patients who had only one visit. Of the remaining 7068 diabetic patients with 2 or more visits during 2011, 975 patients (13.8\%) did not have a documentation of each outcome measure (HbA1c, LDL, and blood pressure) during the 2-year period (2011-2012) and were excluded from the analyses. The final analytic sample is 6111 adult diabetic patients.

The clinic randomization process attempted to balance diabetes structural capabilities, primary care team functioning, and practice size between the two intervention arms and control arm, and did not consider the underlying patient profile [38] of the participating practices given that the modest $\mathrm{CHC}$ clinic sample size precluded use of more than three factors. To address the potential bias of unbalanced data on estimating intervention effects and modest patient sample size that limited direct comparisons of these groups, separate control groups were constructed for the MA and $\mathrm{CHW}$ intervention groups using exact matching on a set of covariates. The exact matching algorithm matched patients on age (10-year bands), sex, race/ethnicity, primary language, and health insurance status (uninsured vs. insured). Matching resulted in at least one match (average of 3.6) per intervention patient. This yielded more valid comparisons between each intervention group and a matched control group for DID regression analyses.

\section{Statistical analyses}

First, we performed a parallel comparison of sociodemographic and health characteristics for each intervention group (MA and $\mathrm{CHW}$ ) and the matched control group. For these unadjusted analyses, we used t-tests to examine differences in continuous measures and $\mathrm{X}^{2}$ tests to examine differences in categorical variables by race/ethnicity and language and compared changes in indicators between intervention and control arms over time. These tests used robust standard errors to handle patient clustering within $\mathrm{CHC}$ sites.

A difference-in-difference (DID) regression approach was used to assess the over-time impact of the $\mathrm{CHW}$ and MA interventions on diabetes care management compared to a matched control group matched on a set of covariates. Multilevel regression models were used to account for the clustering of patients within $\mathrm{CHC}$ sites using site random effects. We compared the binary specifications of HEDIS intermediate outcome measures of LDL-C, HbA1c, and blood pressure. These DID regression models controlled for age, sex, education, self-rated health and insurance status. Due to the collinearity of intervention assignment and racial and ethnic composition of patients at the $\mathrm{CHC}$ sites, the multivariate regression models did not include race/ethnicity as a covariate. To assess the robustness of our estimated intervention effects on diabetes processes and intermediate outcomes, we examine the impact of including patients with incomplete outcome data on diabetes care processes and outcomes as a sensitivity analysis.

A similar regression approach was used for the patient-reported experience and symptom outcome measures. Instead of matching, we included more control variables (patient reported use of insulin, self-rated health) because we had survey and administrative data for this smaller random subgroup of patients. Using the final regression models, we estimated the adjusted estimates of intervention effects for each of the outcome measures (i.e., CG-CAHPS, PACIC-11, hypoglycemia, HbA1c, LDL-C, and blood pressure). Significance was determined using a 2-tailed alpha of 0.05. All analyses were conducted using Stata 14.0.

\section{Interview data analyses}

Transcribed interviews were analyzed using Atlas.ti. We used a qualitative content analysis with a directed approach. [39] A framework of codes was developed by two researchers, and checked for consistency. A log was maintained of emerging codes and used for adapting the coded framework. The adapted framework was reviewed for consistency by two researchers not involved in the coding process. The final list of codes was applied to all interviews by two investigators for each of the key informant roles. Coding discrepancies were reconciled 
during regular research team meetings. Output stratified by intervention group was analyzed and themes were summarized and compared across groups.

\section{Results}

Of the $16 \mathrm{CHC}$ sites that were initially randomized into the intervention and control groups, 2 sites ( 1 from the control group and 1 from the MA intervention group) dropped out of the research study during the early intervention period (January-February 2012) because of data reporting challenges that prevented them from fully participating.

\section{Sample characteristics}

Patients in the MA study arms were older and more likely to be uninsured and of Asian descent compared to $\mathrm{CHW}$ arm patients. Patients of the CHW study arms were more likely to be of Latino descent, had higher BMI levels, and were more likely to have diagnosed and documented mental health co-morbidities, e.g., depression and anxiety, than MA intervention arm patients. The exact matching method balanced patient age, race/ ethnicity, between each intervention group and its separately constructed control group (Table 2).

\section{Clinical process and intermediate outcome results}

In adjusted DID analyses, patients in the CHW intervention arm had improved annual glycated hemoglobin testing (18.5\% points, $p<0.001)$, but the differences were no different than improvements in HbAlc testing among patients in the CHW control group (Table 3). LDL-C testing, however, improved significantly more over time for the CHW control group compared to the CHW intervention group ( $9.8 \%$ points vs. $0 \%, p<0.01$ ).

The MA intervention group patients improved LDL-C control $(8.4 \%$ points, $p<0.05)$ over time, but the improvements were no different than patients in the MA control group (Table 4). HbA1c testing, however, improved more for the MA control group compared to the MA intervention group (5.8\% points vs. $1.0 \%$ points, $p<0.05)$. There were no other differential changes over time for the other diabetes care processes or intermediate outcomes for the MA intervention and control group arms. The final regression results were generally consistent in sensitivity analyses that included patients with one or more missing test values during the study period (data not shown).

\section{Patient-reported measure results}

There were no differential changes in the CG-CAHPS Communication and Access to Care composite scores over time across the study arms (Table 5). Patients in the MA intervention arm reported improved chronic care experiences (PACIC-11) $(\beta=7.5, p<0.001)$ and had significantly greater $(p<0.05)$ improvement compared to control group patients.

Control group patients reported fewer symptoms of diabetes-related emotional distress (PAID-5) over time $(\beta=3.4, p<0.05)$, but the improvement was no different than changes reported by MA or $\mathrm{CHW}$ intervention patients. Self-reported hypoglycemic events did not differentially change over time by study arm.

\section{Key informant interview results}

The key informant interviews of control group $\mathrm{CHC}$ site directors revealed the positive influence of data collection activities associated with project participation on improved documentation, outreach, and in-reach efforts for diabetes care management. This may be one reason why patients of MA control group sites improved HbAlc testing and CHW control sites improved LDL-C testing during the intervention year.

Even though MAs and CHW roles placed different emphases on panel management vs. health coaching in their training, all new personnel in the intervention sites considered health coaching to be their most important job responsibility. In the intervention clinics, MAs and CHWs performed similar duties as health coaches for patients with diabetes, including providing patient education, goal setting, action planning, and routine monitoring. MAs and CHWs reflected on the valuable training they received to implement the new team approaches: "As we were taught to do in training, forcing is not a good way to achieve the goals. Most of the time, I will let patients make the decision". The key informants in the control clinics also considered health coaching to be a very important skill, but all acknowledged that their clinics had not implemented any health coaching responsibilities for staff.

Post-implementation interviews revealed that the project personnel were fully integrated into the diabetes care management workflow. Nevertheless, the MA intervention sites were challenged by the fact that MAs did not have enough dedicated time to conduct health coaching activities because regular MA responsibilities often "crowded out" their less time sensitive health coaching responsibilities. The hectic schedule of floor MA duties may interfere with the health coaching, especially on days when demand is high and staffing is low. As one MA indicated, "Sometimes clinic demands interfere- like we need to help out the clinic flow, or when we are short on manpower. Sometimes, a clinic colleague can't make it that day; then we need to follow doctors to cover for the regular MAs' jobs". Two of the three CHW intervention sites used the CHW personnel as health coaches in full-time positions with unique and separated roles from other primary care personnel. This minimized CHWs from being used to cover for missing 
Table 2 Adult diabetic patient characteristics for the overall and analytic samples, 2011-2013

\begin{tabular}{|c|c|c|c|c|c|}
\hline $\begin{array}{l}\text { Patient characteristic (\% or Mean } \\
\text { and SD) }\end{array}$ & $\begin{array}{l}\text { Overall } \\
\text { sample }\end{array}$ & $\begin{array}{l}\text { Community Health } \\
\text { Worker (CHW) Arm }\end{array}$ & $\begin{array}{l}\text { CHW control } \\
\text { group arm }\end{array}$ & $\begin{array}{l}\text { Medical Assistant } \\
\text { (MA) Arm }\end{array}$ & $\begin{array}{l}\text { MA control } \\
\text { group arm }\end{array}$ \\
\hline $\mathrm{n}$ & 6111 & 686 & 2466 & 644 & 2315 \\
\hline Male (\%) & 41.9 & 30.3 & 30.3 & 47.8 & 47.8 \\
\hline \multicolumn{6}{|l|}{ Age (\%) } \\
\hline $26-35$ & 3.0 & 8.5 & 8.5 & 2.2 & 2.2 \\
\hline $36-45$ & 9.1 & 16.2 & 16.2 & 7.3 & 7.3 \\
\hline $46-55$ & 20.5 & 26.5 & 26.5 & 22.4 & 22.4 \\
\hline $56-65$ & 32.2 & 24.9 & 24.9 & 38.2 & 38.2 \\
\hline $65-75$ & 20.4 & 17.9 & 17.9 & 19.9 & 19.9 \\
\hline 75+ (reference) & 14.9 & 6.0 & 6.0 & 10.1 & 10.1 \\
\hline \multicolumn{6}{|l|}{ Race/Ethnicity/Language (\%) } \\
\hline Asian- Asian language speaking & 49.8 & 1.2 & 1.2 & 72.8 & 72.8 \\
\hline Asian- English speaking & 6.0 & 7.3 & 7.3 & 11.2 & 11.2 \\
\hline Latino- English speaking & 8.2 & 20.1 & 20.1 & 3.0 & 3.0 \\
\hline Other English speaking (reference) & 7.3 & 9.8 & 9.8 & 4.2 & 4.2 \\
\hline Latino- Spanish speaking & 28.7 & 61.7 & 61.7 & 8.9 & 8.9 \\
\hline Uninsured (\%) & 31.9 & 16.0 & 16.0 & 53.3 & 53.3 \\
\hline \multicolumn{6}{|l|}{ Body-Mass Index (BMI) (\%) } \\
\hline BMI (Normal, BMI $\leq 25$ ) (reference) & 27.6 & 12.5 & 13.4 & 39.0 & 39.1 \\
\hline BMI (Overweight, BMI $\geq 26$ and $\leq 30$ ) & 32.9 & 31.3 & 39.4 & 16.3 & 16.1 \\
\hline BMI (Obese I \& II, BMI $\geq 31$ and $\leq 40$ ) & 5.8 & 8.8 & 11.3 & 2.6 & 2.8 \\
\hline BMI (Obese III, BMI 41)) & 24.9 & 29.9 & 26.3 & 37.0 & 35.3 \\
\hline Missing BMl & 8.8 & 17.5 & 9.6 & 5.1 & 6.7 \\
\hline \multicolumn{6}{|l|}{ Co-Morbidities } \\
\hline Cardiovascular Disease (\%) & 8.2 & 5.7 & 6.8 & 6.2 & 7.4 \\
\hline Respiratory Problems (\%) & 7.7 & 8.9 & 8.6 & 4.0 & 5.3 \\
\hline Mental Health (\%) & 13.9 & 22.2 & 20.0 & 7.1 & 8.5 \\
\hline \multicolumn{6}{|c|}{ Number of CHC Visits During the Study Period (\%) } \\
\hline$<=3$ & 8.2 & 6.2 & 9.1 & 7.3 & 10.3 \\
\hline $4-8$ & 39 & 37.6 & 34.9 & 51.9 & 45.8 \\
\hline $9-11$ & 24.5 & 26.3 & 24.3 & 24.1 & 24.4 \\
\hline $12+$ & 28.3 & 32.9 & 31.7 & 16.8 & 19.5 \\
\hline
\end{tabular}

clinic staff. The CHW health coaches were more satisfied with their dedicated time, which allowed them to build expertise and become specialists in their job without feeling peer pressure, compared to the MA intervention group personnel.

\section{Discussion}

The typical 15-min primary care office visit is widely considered insufficient for meeting the educational and treatment needs of vulnerable patients with diabetes and other chronic conditions. Our study aimed to assess the relative impact of routine implementation of two team-based models that aimed to increase patient contact and support improved diabetes care through health coaching and panel management in CHCs. We hypothesized that teams that included CHWs would more effectively improve intermediate outcomes of diabetes, while MAs would be instrumental in improving processes of care. Diabetes care improved in CHCs integrating CHWs and MAs, but neither team-based approach consistently improved both diabetes process or outcome measures. Our results indicate that the intervention groups modestly improved different intermediate outcomes relative to the control groups, i.e., blood pressure control for CHWs and LDL-C control for MAs.

Our key informant interviews results provide important context for these findings, as new roles and responsibilities were found to be nearly identical for MAs and 


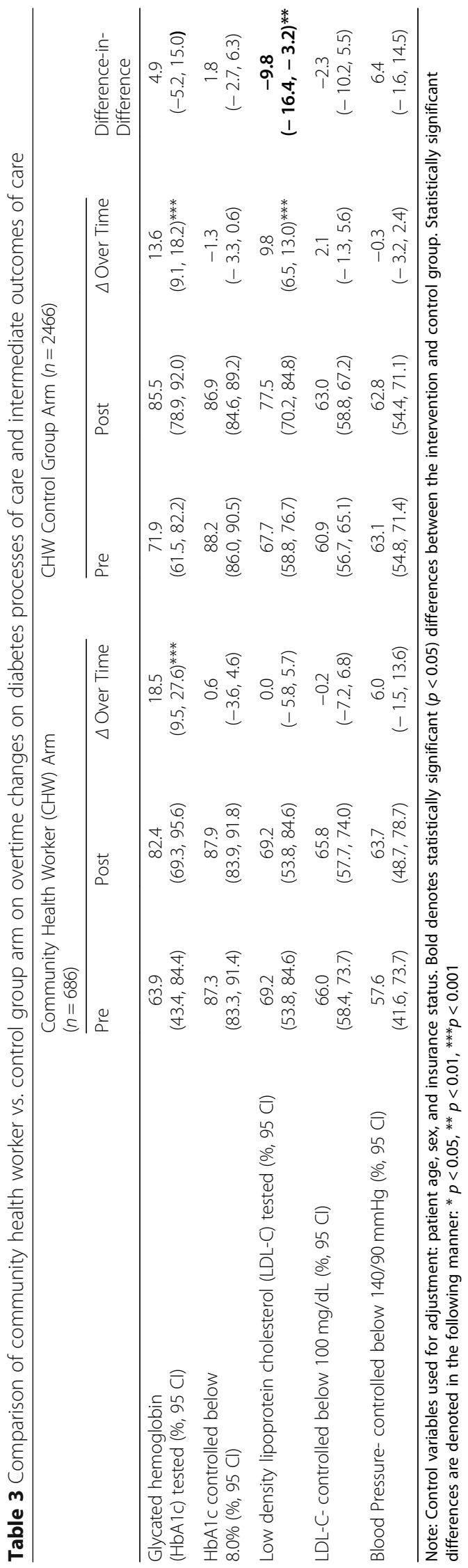




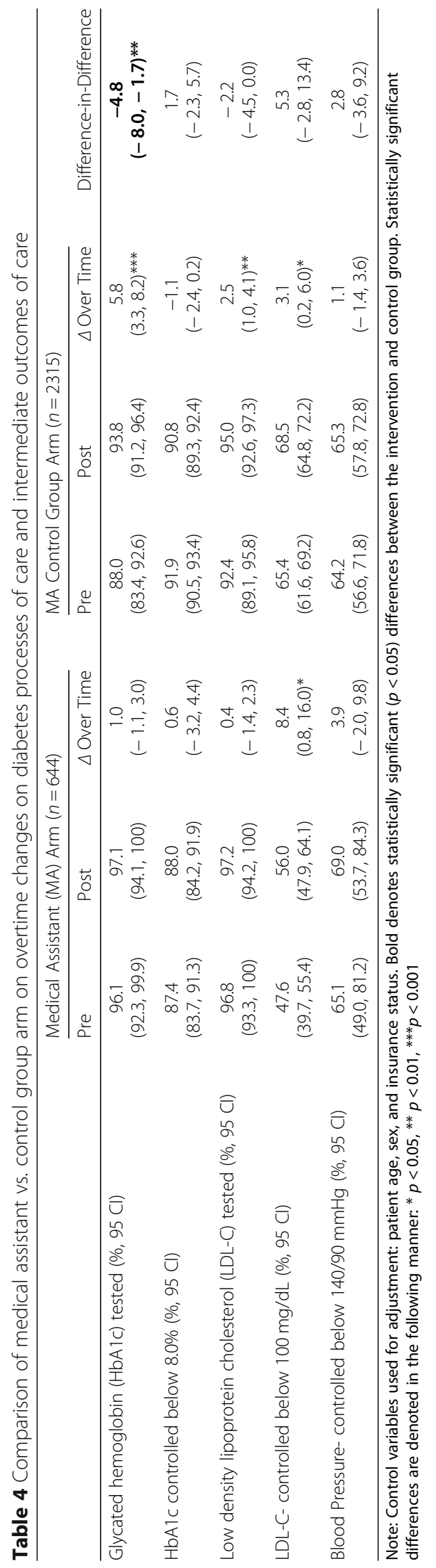




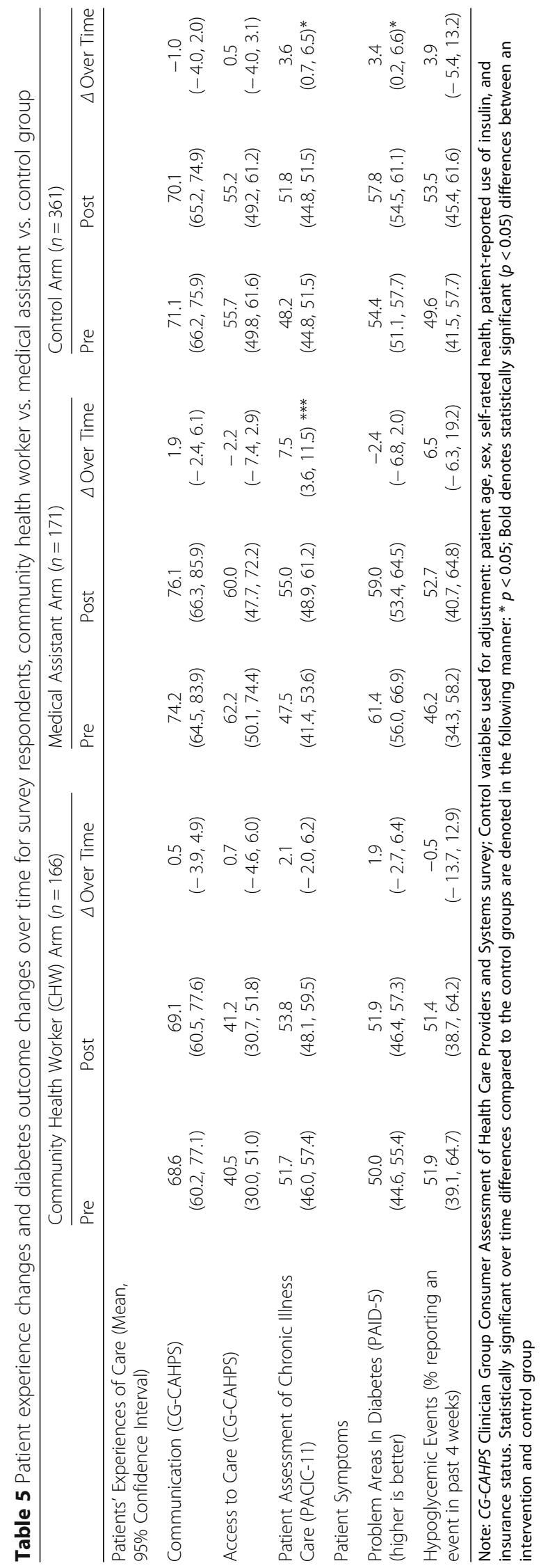


CHWs, with all sites having a high emphasis on health coaching responsibilities. Given the common emphasis patient care responsibilities for new members across clinics and the common MA backgrounds of most intervention personnel, the intervention effects on improved LDL-C control for patients of the MA arm may be a function of the quality improvement of $\mathrm{CHC}$ sites rather than a reflection of generalizable benefits of one model on specific intermediate outcomes of care. Capacity building required of $\mathrm{CHC}$ participation may "contaminate" the study by improving control clinic documentation and care processes, but process improvements did not lead to improved intermediate outcomes for patients of control group clinics. To our surprise, there were negative DIDs for Hbalc testing among the MA group and LDL-C testing among the CHW group, which could not be explained by our key informant interviews and observations.

We anticipated small improvements in patients' experiences of care for the MA and $\mathrm{CHW}$ intervention groups. There were no differential changes in patient experiences between intervention groups and the control group, except for better experiences of chronic care among patients in the MA intervention arm. There is some concern that expanded use of team-based models of primary care will erode PCC and patient relationships because of care coordination and team communication challenges associated with shared care arrangements. While patients' experience scores were generally low and few improvements were observed, it is reassuring that the stressful organizational change of integrating new team roles and responsibilities did not disrupt existing relationships or patients' experiences of access to care.

Our results should be considered with some important limitations in mind. First, we were unable to control for race/ethnicity in regression model due to collinearity issues. Future cluster randomized trials of CHCs should include racial/ethnic composition as a cluster variable because of the challenge of disentangling site vs. patient compositional effects in cluster randomized intervention studies. Second, we experienced the challenge of unbalanced data in cluster randomized intervention trials of modest sample size. We partially addressed this methodological challenge through matching, weighting, and statistical adjustment, but we were not able to perfectly match patients on all study variables, including BMI, co-morbidities and $\mathrm{CHC}$ visits, where small non-significant differences were observed. Third, generalizability is limited due to patient sample attrition and survey non-response. The responses rates to the patient surveys, however, are high for multisite patient experience surveys among $\mathrm{CHC}$ patients. Finally, there were substantial pre-intervention differences in the overall quality of diabetes care between the arms based on the practices' underlying patient characteristics that were not ascertained prior to site randomization. This complicated the direct comparison of MA and CHW intervention arms. Because of this design challenge, we constructed separate matched control groups to enable a more valid estimation of intervention effects. Cluster randomized trials with much larger sets of clinics would reduce the risk of unbalanced allocation of clinics to study arms and could be pursued in the future to minimize these potential biases. Finally, our data were not able to clearly elucidate why specific process and outcome measures improved differentially for the CHW vs. MA team-based models. While our key informant interviews identified barriers, a more robust assessment of fidelity of implementation [40] may have better elucidated what contributed to differential intervention effects for the $\mathrm{CHW}$ vs. MA models. Despite these limitations, our study's pragmatic, mixed methods cluster randomized trial design offers important insights about the benefits and challenges of implementing team-based approaches for diabetes care management in resource limited $\mathrm{CHC}$ settings.

In conclusion, while the team-based interventions examined had some effects on improving diabetes care management, competing demands on staff time impeded the routine integration of new roles and responsibilities for managing diabetes care, especially health coaching. Even though CHW and MA team-based models were relatively low-cost personnel, adoption and implementation in routine practice can still be financially challenging for CHCs. Limited reimbursement of non-clinical staff is a major limitation to the dissemination of team-based models that expand the role of these allied health workers to aid patients in diabetes care management. Innovative payment models, such as the Federally Qualified Health Center Alternative Payment Methodology in Oregon [41], which incentives containment of total costs of care, might stimulate the use of team-based diabetes care management models that include CHWs and MAs, as well as peers [42].

\section{Abbreviations}

BMI: Body-mass index; CG-CAHPS: Clinician \& Group - Consumer Assessment of Healthcare Providers and System; CHCs: Community health centers; CHWs: Community health workers; DID: Difference-in-difference; HbA1c: glycated hemoglobin; HEDIS: Healthcare Effectiveness Data Information Set; ICD-9: International Classification of Diseases, Ninth Revision; LDL-C: low-density lipoprotein cholesterol; MAs: Medical assistants; PACIC 11: Patient Assessment of Chronic Illness Care; PAID-5: Problem Areas in Diabetes scale; PCCs: Primary care clinicians; SUPREME-DM: Surveillance, Prevention, and Management of Diabetes Mellitus 


\section{Acknowledgements}

We thank Elena Alcala Guzman, Dolores Alvarado, Kat Contreras, and Kent Imai of the Community Health Partnership of Santa Clara County for expert technical assistance and practice facilitation. The expert training and coaching provided by Thomas Bodenheimer and Amireh Ghorob of the UCSF Center for Excellence in Primary Care was instrumental to the project. We thank Carmela Castellano-Garcia, Vanesscia Cresci, and Val Sheehan of the California Primary Care Association for their support of health center data integration and feedback activities. We thank Phillip Van der Wees and Dahn Lai for their assistance with key informant interviews and Nigel Lo for his support with programming and statistical analyses.

\section{Funding}

The Agency for Healthcare Research and Quality (AHRQ) funded the research project (1R18HS02012001) through the American Recovery and Reinvestment Act (ARRA). Publication made possible in part by support from the Berkeley Research Impact Initiative (BRII) sponsored by the UC Berkeley Library.

\section{Availability of data and materials}

De-identified study data can be requested by contacting the corresponding author with a proposal.

\section{Authors' contributions}

$H R, A V B$, and MWF designed and planned the study. HPR, AVB, XC, and DHR planned and executed the clinical data and patient survey data collection and statistical analyses. HPR, MWF, and XC planned and executed clinician and staff survey data collection and analyses. EAM and HPR planned the key informant interview analyses, as well as planned the intervention in collaboration with clinical partners. HPR and AEM drafted the study protocol. All authors reviewed the draft version, made suggestions and approved the final version.

\section{Ethics approval and consent to participate}

The human research ethics committee at University of California Los Angeles approved the study protocol (IRB\#10-000596). Consent was obtained from all survey and interview participants.

\section{Consent for publication}

Not applicable.

\section{Competing interests}

The authors declare that they have no competing interests.

\section{Publisher's Note}

Springer Nature remains neutral with regard to jurisdictional claims in published maps and institutional affiliations.

\section{Author details}

'Division of Health Policy and Management, School of Public Health, University of California, Berkeley, 50 University Hall, Room 245, Berkeley, CA 94720, USA. ${ }^{2}$ RAND Corporation, Boston, MA, USA. ${ }^{3}$ Department of Health Policy and Management, Fielding School of Public Health, University of California, California, Los Angeles, USA. ${ }^{4}$ UCLA Center for Health Policy Research, Fielding School of Public Health, University of California, California, Los Angeles, USA. ${ }^{5}$ Division of Health Services Management, School of Public Health, University of Maryland, College Park, MD, USA.

Received: 6 March 2018 Accepted: 13 November 2018

Published online: 20 November 2018

\section{References}

1. Gary TL, Batts-Turner M, Yeh HC, Hill-Briggs F, Bone LR, Wang NY, Levine DM, Powe NR, Saudek CD, Hill MN, et al. The effects of a nurse case manager and a community health worker team on diabetic control, emergency department visits, and hospitalizations among urban African Americans with type 2 diabetes mellitus: a randomized controlled trial. Arch Intern Med. 2009;169(19):1788-94.

2. Carter BL, Ardery G, Dawson JD, James PA, Bergus GR, Doucette WR, Chrischilles EA, Franciscus CL, Xu Y. Physician and pharmacist collaboration to improve blood pressure control. Arch Intern Med. 2009; 169(21):1996-2002.
3. Rosenblatt RA, Andrilla CH, Curtin T, Hart LG. Shortages of medical personnel at community health centers: implications for planned expansion. J Am Med Assoc. 2006;295(9):1042-9.

4. Zhuo $X$, Zhang P, Hoerger TJ. Lifetime direct medical costs of treating type 2 diabetes and diabetic complications. Am J Prev Med. 2013;45(3):253-61.

5. Seuring T, Archangelidi $O$ and Suhrcke M. The Economic Costs of Type 2 Diabetes: A Global Systematic Review. Pharmacoeconomics. 2015;33:811831. https://doi.org/10.1007/s40273-015-0268-9.

6. Mensing C, Boucher J, Cypress M, Weinger K, Mulcahy K, Barta P, Hosey G, Kopher W, Lasichak A, Lamb B, et al. National standards for diabetes selfmanagement education. Diabetes Care. 2007;30(Suppl 1):S96-S103.

7. Sarkar U, Piette JD, Gonzales R, Lessler D, Chew LD, Reilly B, Johnson J, Brunt M, Huang J, Regenstein M, et al. Preferences for self-management support: findings from a survey of diabetes patients in safety-net health systems. Patient Educ Couns. 2008;70(1):102-10.

8. Scanlon DP, Hollenbeak CS, Beich J, Dyer AM, Gabbay RA, Milstein A. Financial and clinical impact of team-based treatment for medicaid enrollees with diabetes in a federally qualified health center. Diabetes Care. 2008;31(11):2160-5.

9. O'Brien MJ, Squires AP, Bixby RA, Larson SC. Role development of community health workers: an examination of selection and training processes in the intervention literature. Am J Prev Med. 2009;37(6 Suppl 1):S262-9.

10. Cherrington A, Ayala GX, Amick H, Scarinci I, Allison J, Corbie-Smith G. Applying the community health worker model to diabetes management: using mixed methods to assess implementation and effectiveness. J Health Care Poor Underserved. 2008;19(4):1044-59.

11. Employment Projections: Occupations with the Largest Job Growth [https:// www.bls.gov/emp/tables/occupations-most-job-growth.htm]. Accessed 17 Nov 2018.

12. Bosley S, Dale J. Healthcare assistants in general practice: practical and conceptual issues of skill-mix change. Br J Gen Pract. 2008;58(547):118-24.

13. Tache S, Chapman S. The expanding roles and occupational characteristics of medical assistants: overview of an emerging field in allied health. J Allied Health. 2006;35(4):233-7.

14. Thom DH, Hessler D, Willard-Grace R, DeVore D, Prado C, Bodenheimer T, Chen EH. Health coaching by medical assistants improves patients' chronic care experience. Am J Manag Care. 2015;21(10):685-91.

15. Willard-Grace $R$, Chen EH, Hessler D, DeVore D, Prado C, Bodenheimer T, Thom DH. Health coaching by medical assistants to improve control of diabetes, hypertension, and hyperlipidemia in low-income patients: a randomized controlled trial. Ann Fam Med. 2015;13(2):130-8.

16. MacPhail LH, Neuwirth EB, Bellows J. Coordination of diabetes care in four delivery models using an electronic health record. Med Care. 2009;47(9):993-9.

17. Vargas Bustamante A, Martinez A, Chen X, Rodriguez HP. Clinic workload, the quality of staff relationships and diabetes Management in Community Health Centers Catering to Latino and Chinese patients. J Community Health. 2017:42(3):481-8.

18. Islam N, Nadkarni SK, Zahn D, Skillman M, Kwon SC, Trinh-Shevrin C. Integrating community health workers within patient protection and affordable care act implementation. J Public Health Manag Pract. 2015; 21(1):42-50.

19. Shah MK, Heisler M, Davis MM. Community health workers and the patient protection and affordable care act: an opportunity for a research, advocacy, and policy agenda. J Health Care Poor Underserved. 2014;25(1):17-24.

20. Cherrington A, Ayala GX, Amick H, Allison J, Corbie-Smith G, Scarinci I. Implementing the community health worker model within diabetes management: challenges and lessons learned from programs across the United States. Diabetes Educ. 2008;34(5):824-33.

21. Heisler M, Spencer M, Forman J, Robinson C, Shultz C, Palmisano G, GraddyDansby G, Kieffer E. Participants' assessments of the effects of a community health worker intervention on their diabetes self-management and interactions with healthcare providers. Am J Prev Med. 2009;37(6 Suppl 1):S270-9.

22. Weiler DM, Crist JD. Diabetes self-management in a Latino social environment. Diabetes Educ. 2009;35(2):285-92.

23. Palmas W, March D, Darakjy S, Findley SE, Teresi J, Carrasquillo O, Luchsinger JA. Community health worker interventions to improve glycemic control in people with diabetes: a systematic review and meta-analysis. J Gen Intern Med. 2015;30(7):1004-12.

24. Rodriguez HP, Rogers WH, Marshall RE, Safran DG. Multidisciplinary primary care teams: effects on the quality of clinician-patient interactions and organizational features of care. Med Care. 2007;45(1):19-27. 
25. Curran GM, Bauer M, Mittman B, Pyne JM, Stetler C. Effectiveness-implementation hybrid designs: combining elements of clinical effectiveness and implementation research to enhance public health impact. Med Care. 2012;50(3):217-26.

26. Friedberg MW, Coltin KL, Safran DG, Dresser M, Schneider EC. Medical home capabilities of primary care practices that serve sociodemographically vulnerable neighborhoods. Arch Intern Med. 2010;170(11):938-44.

27. Friedberg MW, Rodriguez HP, Martsolf GR, Edelen MO, Vargas Bustamante A. Measuring workplace climate in community clinics and health centers. Med Care. 2016;54(10):944-9.

28. Ngo V, Hammer H, Bodenheimer T. Health coaching in the teamlet model: a case study. J Gen Intern Med. 2010;25(12):1375-8.

29. Rodriguez HP, Crane PK. Examining multiple sources of differential item functioning on the Clinician \& Group CAHPS(R) survey. Health Serv Res. 2011:46(6pt1):1778-802.

30. Gugiu PC, Coryn C, Clark R, Kuehn A. Development and evaluation of the short version of the patient assessment of chronic illness care instrument. Chronic IIIn. 2009;5(4):268-76.

31. McGuire BE, Morrison TG, Hermanns N, Skovlund S, Eldrup E, Gagliardino J, Kokoszka A, Matthews D, Pibernik-Okanovic M, Rodriguez-Saldana J, et al. Short-form measures of diabetes-related emotional distress: the problem areas in diabetes scale (PAID)-5 and PAID-1. Diabetologia. 2010;53(1):66-9.

32. Sarkar U, Karter AJ, Liu JY, Moffet HH, Adler NE, Schillinger D. Hypoglycemia is more common among type 2 diabetes patients with limited health literacy: the diabetes study of northern California (DISTANCE). J Gen Intern Med. 2010;25(9):962-8.

33. Quinn MT, Gunter KE, Nocon RS, Lewis SE, Vable AM, Tang H, Park SY, Casalino LP, Huang ES, Birnberg J, et al. Undergoing transformation to the patient centered medical home in safety net health centers: perspectives from the front lines. Ethn Dis. 2013;23(3):356-62.

34. Aragones A, Schaefer EW, Stevens D, Gourevitch MN, Glasgow RE, Shah NR. Validation of the Spanish translation of the patient assessment of chronic illness care (PACIC) survey. Prev Chronic Dis. 2008;5(4):A113.

35. Nunnelly J, Bernstein I. Psychometric theory. New York: McGraw-Hill; 1994.

36. Van der Wees PJ, Friedberg MW, Guzman EA, Ayanian JZ, Rodriguez HP. Comparing the implementation of team approaches for improving diabetes care in community health centers. BMC Health Serv Res. 2014;14:608.

37. Nichols GA, Desai J, Elston Lafata J, Lawrence JM, O'Connor PJ, Pathak RD, Raebel MA, Reid RJ, Selby JV, Silverman BG, et al. Construction of a multisite DataLink using electronic health records for the identification, surveillance, prevention, and management of diabetes mellitus: the SUPREME-DM project. Prev Chronic Dis. 2012;9:E110.

38. Johnson ML, Rodriguez HP, Solorio MR. Case-mix adjustment and the comparison of community health center performance on patient experience measures. Health Serv Res. 2010;45(3):670-90.

39. Hsieh HF, Shannon SE. Three approaches to qualitative content analysis. Qual Health Res. 2005;15(9):1277-88.

40. Grace SM, Rich J, Chin W, Rodriguez HP. Fidelity of implementation to a care team redesign and improved outcomes of diabetes care. Int J Qual Health Care. 2015;27(1):60-6.

41. Cottrell EK, Hall JD, Kautz G, Angier H, Likumahuwa-Ackman S, Sisulak L, Keller S, Cameron DC, DeVoe JE, Cohen DJ. Reporting from the front lines: implementing Oregon's alternative payment methodology in federally qualified health centers. J Ambul Care Manage. 2017;40(4):339-46.

42. Kaselitz E, Shah M, Choi H, Heisler M. Peer characteristics associated with improved glycemic control in a randomized controlled trial of a reciprocal peer support program for diabetes. Chronic illness. 2018;1742395317753884. https://doi.org/10.1177/1742395317753884.

Ready to submit your research? Choose BMC and benefit from:

- fast, convenient online submission

- thorough peer review by experienced researchers in your field

- rapid publication on acceptance

- support for research data, including large and complex data types

- gold Open Access which fosters wider collaboration and increased citations

- maximum visibility for your research: over $100 \mathrm{M}$ website views per year

At BMC, research is always in progress.

Learn more biomedcentral.com/submissions 